\title{
Association of Dietary w-6/w-3 Fatty Acid Ratio and Inflammation with Risk of Hip Fracture
}

\author{
Kumar Kartikey ${ }^{1, *}$, Garima Singh ${ }^{1}$, Brajesh Kidyore ${ }^{1}$, Y A Somsunder ${ }^{1}$, P. Howarth ${ }^{2}$, \\ Viola Vargova ${ }^{2}$, Zelmira Macejova, Fabien ${ }^{2}$ De Meester ${ }^{3}$ and R.B. Singh ${ }^{3}$ \\ ${ }^{I}$ Department of Orthopedics, Sidhartha Medical College, Tumkur (AP), India \\ ${ }^{2}$ Faculty of Medicine, Safaric University, Slovakia \\ ${ }^{3}$ Tsim Tsoum Institute, Krakow, Poland
}

\begin{abstract}
Objectives: There is evidence that calcium, magnesium, vitamin D, proteins, antioxidants and w-3 fatty acids intakes are inversely associated with risk of osteoporosis and hip fractures. In the present study we examine the association of food consumption pattern and w- $6 / \mathrm{w}-3$ fatty acid ratio of the diet with hip joint fractures.

Methods: Sixty cases having fracture neck of femur and 95 control subjects above 50 years of age were included in this case control study. Dietary intakes were obtained by 3 days assessment of food intakes by questionnaires among patients with fractures $(\mathrm{n}=50)$ as well as among 95 control subjects. Physical activity was assessed by questionnaires. Radiological and clinical examination were conducted for the diagnosis of fractures. Cytokines were measured by chemoluminescence enzyme immunometric assay (immulite automated analyzer) kit (DPC Los Angelis, CA, USA). Regression analysis was done to find out the association of risk factors with hip fractures.

Results: Among 60 cases, the fracture was more common in male than female. Fruits, vegetables and legume(165 \pm 12.6 vs. $205 \pm 15.8 \mathrm{~g} /$ day, $\mathrm{P}<0.03)$ as well as milk products (milk, curd, butter etc) consumption $(205+25.8$ vs. $318 \pm 31.5 \mathrm{~g} /$ day, $\mathrm{P}<0.05$ ) were significantly lower and $\mathrm{w}-6$ rich oils intake was significantly higher among patients with fractures compared to control subjects, respectively. Omega-3 fatty acids intakes were significantly lower among patients with fractures $(0.45 \pm 0.74 \mathrm{~g}$ /day, $\mathrm{P}<0.05)$. Osteoporosis $(92.0 \%)$, trivial trauma $(92.0 \%)$, physical inactivity $(80.0 \%)$, diabetes mellitus $(21.6 \%)$ were common among patients with hip fracture. Multivariate logistic regression analysis showed that the intakes of fruit, vegetable and legume (odds ratio 1.12, confidence interval (CI) 1.02-1.21, $\mathrm{P}<0.05$ ), physical activity(OR 1.36, CI 1.22-1.52, $\mathrm{P}<0.05)$, w-3 fatty acids (OD1.05, 0.92-1.17, $\mathrm{P}<0.01)$ intake were inversely associated with fracture, whereas w-6/w-3 ratio (OD 1.33, CI 1.18-1.47,P<0.01) interleukin-6, (OD1.11, CI 1.02-1.19, $\mathrm{P}<0.01$ ), tumor necrosis factor-alpha(OD,1.09, CI 1.01-1.17, $\mathrm{P}<0.01)$ were positively associated with fracture.
\end{abstract}

Conclusion: This study showed that increased consumption of fruit, vegetable and legume, milk products and w-3 fatty acid and low w-6/w-3 ratio diet as well as physical activity may be protective against hip joint fractures.

Keywords: Nutrition, dietary pattern, fatty acids, inflammation, bone disease, cytokines.

\section{INTRODUCTION}

There is convincing evidence for a reduction in risk for osteoporosis with increased intake of vitamin D and calcium and for an increased risk with high intake of alcohol and undernutrition. No study has examined the role of nutrients among post operative outcome in patients with hip fracture. Dietary calcium, vitamin D have been studied in the pathogenesis of osteoporosis which is known to predispose fractures. However, the role of w-3 fatty acid metabolism in the pathogenesis of osteoporosis has not been studied thoroughly. Osteoporosis is a disease affecting many millions of people around the world. It is characterized by low bone

*Address correspondence to this author at the (Orthopedics), FICN, Senior Resident, Teerthankar Medical College, Halberg Hospital and Research, Institute, Civil Lines, Moradabad- 10 (UP) 244001, India;

Tel/Fax: 0091591 2417437; E-mail: drkk@dataone.in mass and micro-architectural deterioration of bone tissue, leading to bone fragility and a consequent increase in risk of fracture $[1,2]$. The incidents of vertebral and hip fractures increases exponentially with advancing age (while that of risk of fractures levels off after the age of 60 years [3]. Osteoporosis fractures are a major cause of morbidity and disability in older people and, in the case of hip fractures can lead to pre-mature death. Such fractures impose a considerable economic burden on health services worldwide [4]. Worldwide variation in the incident and prevalence of osteoporosis is difficult to determine because of problems with definition and diagnosis. The most usefull way of comparing osteoporosis prevalence between populations is to use fracture rates in older people. However, because osteoporosis is usually not life threatening, quantitative data from developing countries are scarce. despite this, the current consensus is that approximately 1.66 million hip fractures occur each year worldwide, that the incidence is set to increase four-fold by 
2050 because of the increasing numbers of older people, and that the age -adjusted incidence rates are many times higher in affluent developed countries than in sub Saharan Africa and Asia [1-4].

The incidence of hip fractures in both sexes increases exponentially with age. Hip fractures rates are highest in Caucasian women living in temperate climate, are somewhat lower in women from Mediterranian and Asian countries, and are lowest in women in Africa [1-4]. The lower incidence of hip fractures in these countries indicate that differences in diet and lifestyle appear to be important in the pathogenesis and prevention of osteoporosis and hip fractures [5-13]. Experimental studies [6-8] indicate that the high intake of w-6 and inadequate amount of w- 3 fatty acids in the diet contribute to the development of several pathologies of bone and joint diseases including osteoporosis which is a major cause of hip fractures. Recently, increased intake of fruits, vegetables, legumes and nuts that are rich in w-3 fatty acids (alpha linolenic acid) have been found protective against risk of osteoporosis, which poses the possibility that these foods and nutrients may protect against fracture and provide better quality of life [5-11]. This study reports on the association of w-6/w-3 fatty acid ratio of the diet and inflammatory cytokines with risk of hip fractures.

\section{SUBJECTS AND METHODS}

The present study consists of 60 patients with traumatic fracture neck of femur, and 95 apparently health subjects $>50$ years of age attending the Sree Siddhartha Medical College, Tumkur and District Hospital, Tumkur, South India, between October 2003 to October 2005. All the cases taken up for this study gave informed consent to participate in the study and were fresh fractures, admitted to our hospitals. The study was approved by the ethic committee of the college. A surgeon administered the questionnaire to obtain information on age, sex, physical activity, tobacco intake and alcohol intake. Physical examination included measurement of height and weight. Body weight was measured independently by the surgeon in light underclothes nearest of $0.5 \mathrm{Kg}$. Subjects who admitted to drinking alcohol at least once per week were categorized as alcohol consuming and $>10$ drinks per week as alcoholic.. Physical activity was assessed by questionnaire based on occupational, household and spare time activity [12]. The criteria for sedentary lifestyle was based on walking $<14 \mathrm{Km}$ a week or climbing fewer than 20 flights of stairs a week during household or occupational activities in absence of moderately vigorous spare time physical activity on five days a week.

Dietary intakes were obtained by 3 days assessment of food intakes by validated food intake questionnaires, among patients with fractures $(n=60)$ as well as among 95 control subjects above 50 years of age. Food measures, food portions and food models were used during the interview of the patients and the subjects, to find out exact quantity of foods filled in the questionnaires. Nutrient intakes were calculated with the help of Indian food composition tables (13). Radiological and clinical examinations were conducted for the confirmation of the diagnosis of fractures. Biochemical data were obtained by standard methods using chemical kit from USA. Fasting blood sample were obtained after 12 hours of fast in the morning for analysis of biochemical data in all the patients and subjects in both the groups.Thiobarbituric acid reactive substances(TBARS), melondialdehyde(MDA) (quatitatively) were obtained by colorimetric methods. MDA was quantified by a reaction with thiobabituric acid $[14,15]$.

Cytokines; interleukin (IL)-6, and tumor necrosis factor (TNF)-alpha were determined by using a solid phase, two sided chemoluminescence enzyme immunometric assay (immulite automated analyzer) kit (DPC Los Angelis, CA,USA). The plasma samples were kept at -4 o C, until the assay was performed. Results were expressed as $\mathrm{pg} / \mathrm{ml}$.

\section{RESULTS}

The age ranged from $60-82$ years, predominantly $60-65$ years $(n=40$ cases, $66 \%)$. There were $40(66 \%)$ men and 20 $(33 \%)$ women. Sex differences in various data were not observed. In half of the patients, $(n=30)$ trivial fall was the commonest mode of injury while walking inside or outside of the house.

Risk factors of fractures were, postmenopausal state $(100.0 \%)$, undernutrition (33.3\%), osteoporosis $(92.0 \%)$ trivial trauma (92.0\%) physical inactivity $(80.0 \%)$, diabetes mellitus(21.6\%) and alcoholism (10\%) (Table 1).

Fruits, vegetables and legume $(165 \pm 12.6 v s .205 \pm 15.8 \mathrm{~g} /$ day, $\mathrm{P}<0.03$ ) as well as milk products (milk, curd, butter etc) consumption $(205 \pm 25.8$ vs. $318 \pm 31.5 \mathrm{~g} /$ day, $\mathrm{P}<0.05)$ were significantly lower among patients with fractures compared to control subjects, respectively. Omega-3 fatty acids (alphalinolenic acid) intakes also showed significantly lower intake among patients with fractures compared to healthy subjects ( 0.45 vs $0.74 \mathrm{~g} /$ day, $\mathrm{P}<0.05$ ). The consumption of $\mathrm{w}-$ 6 fatty acid rich oils, vegetable ghee and sugar and syrups were significantly greater among patients than control subjects (Table 2).

Table 3 shows the concentration of proinflammatory cytokines among patients and control subjects. Plasma levels of IL-6, tumor necrosis factor (TNF)-alpha as well as thiobarbituric acid reactive substances and malondialdehyde were significantly greater among patients with fractures compared to control subjects.

Multivariate logistic regression analysis showed that after adjustment of age and sex, the intakes of fruit, vegetable and legume, and w-3 fatty acids intake were inversely associated with fracture, whereas W-6/W-3 fat ratio, sedentary lifestyle, IL-6 and TNF-alpha were positively associated with fracture (Table 4).

\section{DISCUSSION}

This study shows that high w-6/w-3 fat ratio and sedentary lifestyle were significantly associated with risk of fractures, whereas, w-3, and fruit, vegetable and legume intakes were inversely associated with fractures (Table 4). We do not have such studies from India hence we can not compare our findings with other studies. In a single blind, randomized trial [9], the interactions of calcium, dihomogamalinolenic acid (DGLA)+ eicosapentaenoic acid (EPA) was examined compared to coconut oil+calcium in women with osteoporosis. All subjects were living in a institution for elderly and fed low calcium, non-vitamin D enriched foods and similar 
Table 1. Clinical Data among Patients with Hip Fracture and Control Subjects

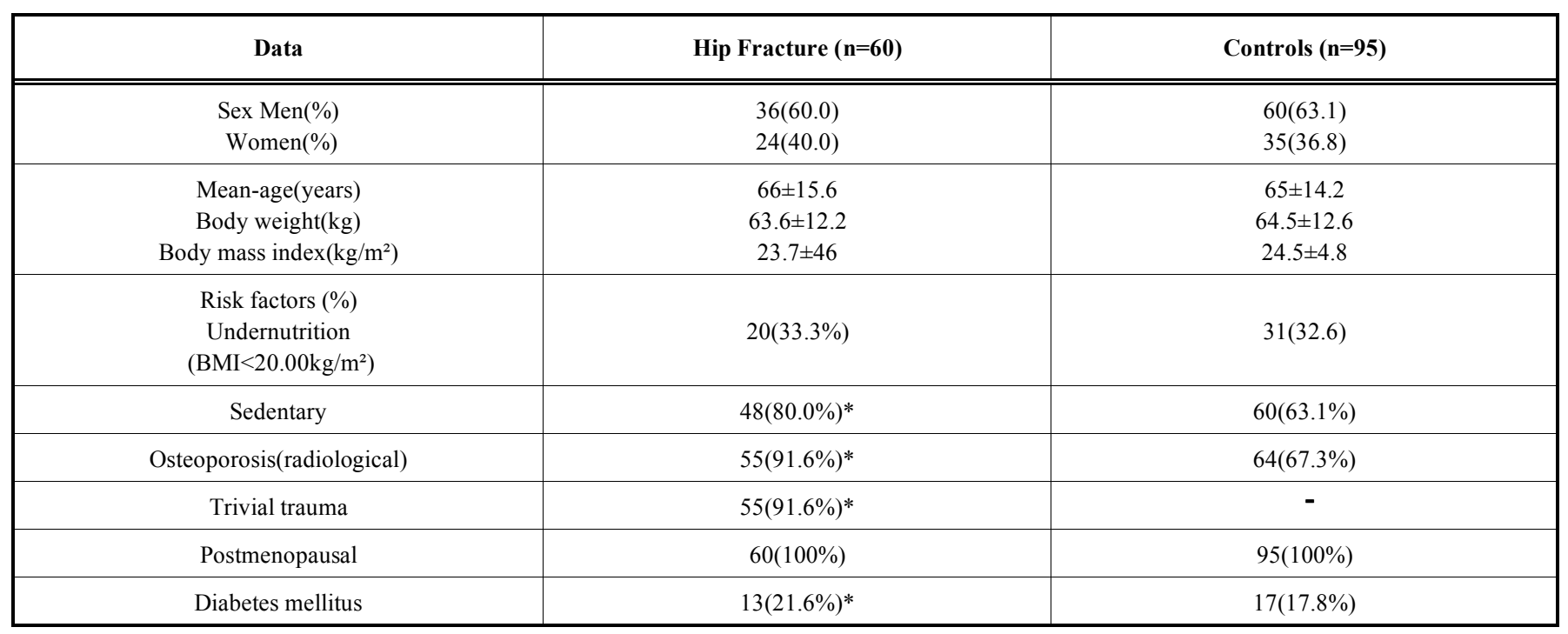

$*=\mathrm{P}<0.05$ by comparison of values among cases with controls.

Table 2. Food Consumption Pattern among Patients of Hip Joint Fracture and Controls

\begin{tabular}{|c|c|c|}
\hline Cereals and grains & $210 \pm 15.5$ & $226 \pm 21.6$ \\
\hline Fruits & $66 \pm 6.7 *$ & $87 \pm 9.9$ \\
\hline Vegetables & $60 \pm 6.2 *$ & $82 \pm 8.7$ \\
\hline Fruits, vegetables and legumes & $165 \pm 12.6^{*}$ & $205 \pm 15.8$ \\
\hline Milk and milk products & $205 \pm 25.8^{*}$ & $318 \pm 31.5$ \\
\hline Total visible fat & $49.3 \pm 5.8 *$ & $50.6 \pm 7.5$ \\
\hline Indian ghee or butter & $4.3 \pm 1.2$ & $4.6 \pm 2.3$ \\
\hline Sugar and jaggery, syrups & $26.6 \pm 8.5^{*}$ & $22.6 \pm 6.5$ \\
\hline Salt & $12.6 \pm 3.4$ & $14.6 \pm 4.7$ \\
\hline Energy, k.cal/day & $1950 \pm 502$ & $2015 \pm 518$ \\
\hline Total carbohydrates (k.cal/day) & $56.2 \pm 8.5$ & $57.0 \pm 9.5$ \\
\hline Total proteins (k.cal/day) & $13.3 \pm 3.4$ & $13.5 \pm 3.7$ \\
\hline Total fat(k.cal/day) & $30.5 \pm 5.7$ & $29.5 \pm 6.1$ \\
\hline Saturated fat(k.cal/day) & $10.5 \pm 3.1$ & $10.0 \pm 2.9$ \\
\hline
\end{tabular}


Table 3. Laboratory Data among Patients and Control Subjects

\begin{tabular}{|c|c|c|}
\hline Data & Patients (n=60) & Controls (n=95) \\
\hline \hline Hemoglobin $(\mathrm{g} / \mathrm{dl})$ & $9.5 \pm 2.3$ & $98.6 \pm 9.8$ \\
\hline Blood glucose $(\mathrm{mg} / \mathrm{dl})$ & $100 \pm 10.2$ & $28.6 \pm 5.2$ \\
\hline Blood urea $(\mathrm{mg} / \mathrm{dl})$ & $30.0 \pm 5.5$ & $10.2 \pm 3.2$ \\
\hline Interleukin-6 $(\mathrm{pg} / \mathrm{ml})$ & $22.5 \pm 4.2^{* *}$ & $11.6 \pm 3.5$ \\
\hline Tumor necrosis factor-alpha $(\mathrm{pg} / \mathrm{ml})$ & $24.6 \pm 4.5^{* *}$ & $1.0 \pm 0.42$ \\
\hline TBARS (pmol/L) & $1.8 \pm 0.55^{*}$ & $2.1 \pm 1.0$ \\
\hline Malondialdehyde $(\mathrm{pmol} / \mathrm{L})$ & $2.8 \pm 1.1^{*}$ & \\
\hline
\end{tabular}

$*=\mathrm{P}<0.05, * *=\mathrm{P}<0.01$.

Table 4. Multivariate Logistic Regression Analysis for Association of Various Risk Factors with Hip Fracture after Adjustment of Age and Sex

\begin{tabular}{|c|c|c|}
\hline Risk Factor & Odd,s Ratio & 95\% Confidence Interval \\
\hline \hline Sedentary behaviour & $1.36^{*}$ & $1.22-1.52$ \\
\hline Fruits, vegetable and legume intake & $-1.12^{*}$ & $1.02-1.21$ \\
\hline Omega-3 fatty acids & $-1.05^{* *}$ & $1.92-1.17$ \\
\hline Omega-6/Omega-3 fat ratio & $1.33^{* *}$ & 1.47 \\
\hline Interleukin-6 & $1.11^{*}$ & $1.02-1.19$ \\
\hline Tumor necrosis factor-alpha & $1.09^{* *}$ & 1.17 \\
\hline
\end{tabular}

$*=\mathrm{P}<0.05, * *=\mathrm{P}<0.01$.

exposure to sunlight. Markers of bone formation or degradation and bone mineral density (BMD) were measured at baseline, 6, 12 and 18 months duration. After 18 months, osteocalcin and deoxypyridinoline levels decreased significantly in both groups, indicating a reduction in bone turnover, whereas bone specific alkaline phosphatase increased indicating beneficial effects of calcium given to all the patients. Lumber and femoral BMD showed that lumber spine density remained the same in the treatment group, but decreased $3.2 \%$ in the placebo group. Femoral bone density increased $1.3 \%$ in the treatment group but decreased $2.1 \%$ in the placebo group. During the second period of 18 months with all women receiving intervention treatment, lumber spine density increased $3.1 \%$ among patients who remained on active intervention and $2.3 \%$ among those women who switched from placebo to active treatment; femoral BMD in the latter group showed an increase of $4.7 \%$.

In another study by Terano [10], 40 elderly women with age related osteoporosis were divided into four groups. They were administered daily for 16 weeks one of 4 treatment regimens in each group; $4 \mathrm{~g}$ evening primrose oil, $4 \mathrm{~g}$ fish oil, $4 \mathrm{~g}$ of a fish and evening primrose oil mixture or $4 \mathrm{~g}$ olive oil placebo, while no other medication/ supplements or special foods was taken during the trial. In this study, fish oil increased serum calcium, osteocalcin and collagen and decreased ALP. Evening primrose oil alone had no significant effects, but the positive results from the fish oil group were also seen in the fish oil plus evening primrose oil group. It is possible that evening primrose oil may have synergestic effects with fish oil. Evening primrose oil also contains ALA which is metabolized in our body into EPA and docosahaxaenoic acid (DHA) that are long chain w-3 fatty acids present in fish oil.

There is evidence that depression may be associated with reduced BMD [11]. In one study [11] 24 women with a history of major depression were compared with another 24 age, race and body mass index, postmenopausal status matched women controls. There was a significant lower BMD in patients with depression compared to controls. Deficiency of w-3 fatty acids may be a common link between depression and osteoporosis among these subjects, both prevalent among elderly population. These studies [9-10] indicate that $\mathrm{w}-3$ fatty acids appear to be important in bone health and in the prevention of osteoporosis and support our findings that increased ratio of $\mathrm{w}-6 / \mathrm{w}-3$ fatty acids or deficiency of w-3 fatty acids may be a risk factor for osteoporosis and hip fractures (Table 4).

Further support for the role of polyunsaturated fatty acids in the pathogenesis of osteoporosis and hip fracture may be provided from the experimental studies [16-18]. In a experimental study [16], 15 rats were fed identical diets except that w-6/w-3 ratio differed. Safflower oil and fish oil were mixed 
to produce $\mathrm{w}-6 / \mathrm{w}-3$ ratios of $23.8,9.8,2.6$ and 1.2. There was a significant increase in PGE2 and ALP with decrease in $\mathrm{w}-6 / \mathrm{w}-3$ ratio in the rat liver and bone tissue [16]. The high concentration of ALP indicated better reabsorption of bone and moreover $1.2 \mathrm{w}-6 / \mathrm{w}-3$ ratio diet showed higher rate of bone formation. In another study [17], an increased production of PGE2 in tibia of chicks given a semipurified diet containing soya bean oil, high in w-6 fat was associated with a lower rate of bone formation compared with that of chicks fed a low dietary ratio of $w-6 / w-3$ fatty acids. In a further study [18], rats fed with a lower dietary ratio of w-6/w-3 fat showed increased bone marrow cellularity and bone strength. These studies indicate that w-6/w-3 fatty acid ratio of diet appears to be important in bone metabolism, and may be responsible for fracture due to trivial trauma in our patients.

These studies provide further proof that dietary fatty acids modulate the fatty acid composition and PGE2 production in these tissues by altering fatty acid composition of membrane phospholipids resulting into improvement in cell function. The bone is highly active metabolic tissue, that continues to change throughout life. Bone remodeling occurs upto $20 \%$ which is the process of bone growth associated with maintaining a fixed adult bone mass [6-11]. Older bone tissue is destroyed (reabsorbed) and replaced by new bone tissue in a cyclical process. However, in osteoporosis, the basic cause is that reabsorption becomes greater ahead of bone formation, resulting in a net bone loss. Diets higher in w-6/w-3 fatty acids are associated with greater PGE2 which have adverse effects [16-18]. Whereas low $\mathrm{w}-6 / \mathrm{w}-3$ ratio diet may provide low PGE2 which stimulates bone formation by increased production of insulin like growth factor, which is a powerful growth stimulator for bone and muscle. The growth may be further enhanced by simultaneous treatment with coenzyme Q10 which is potent antioxidant and bioenergetic agent present in the mitochondria of muscles. PGE2 also mediate the effects of vitamin D, TNF-alpha and growth factors which are known to enhance bone reabsorption which results into osteoporosis [16-18].There was a positive association of proinflammatory cytokines; IL-6 and TNF-alpha in patients with hip fracture, in presence of w-3 fatty acids deficiency in the diet (Table 3 and 4). Therefore it is possible that w-3 fatty acids modulates PGE2 as well as proinflammatory cytokines; TNF-alpha and IL-6 which are known to enhance PGE2 [19], resulting in to marked increase in bone reabsorption.

In brief, the findings of our study indicate that higher w6/w-3 fat diet, lower intake of fruits, vegetables and legumes and sedentary behaviour may be associated with hip fractures, and possibly osteoporosis. It is possible that increased consumption of these foods along with low w-6/ w-3 fatty acids diet and moderate physical activity, may be protective against hip fractures.

\section{REFERENCES}

[1] Lau EM, Coopper C. The epidemiology of osteoporosis: the oriental perspective in a world context. Clin Orthop Relat Res 1996; 323: 65-74.

[2] Cooper C, Campion G, Melton LJ. Hip fractures in the elderly: a world-wide projection. Osteoporos Int 1992; 2: 285-9.

[3] Johnell C. The socioeconomic burden of fractures: today and in the $21^{\text {st }}$ century. Am J Med 1997; 103 (Suppl 2A): 20-5.

[4] Report of a Joint WHO/FAO Expert Consultation. Diet, Nutrition and the Prevention of Chronic Diseases. WHO, Geneva 2003; pp. 129-33.

[5] Sullivan DH, Nelson CL, Klimberg VS, Bopp MM. Nightly enteral nutrition support of elderly hip fracture patientsd: a pilot study. J Am Coll Nutr 2004; 23: 683-91.

[6] Watkins BA. Regulatory effects of polyunsaturates on bone remodeling and cartilage function. World Rev Nutr Diet 1998; 83: 38-51.

[7] Watkins BA, Seifert MF, Allen KG. Importance of dietary fat in modulating PGE2 responses and influence of vitamin $\mathrm{E}$ on bone morphometry. World Rev Nutr Diet 1997; 82: 250-9.

[8] Watkins BA, Seifert MF. Conjugated linoleic acid and bone biology. J Am Coll Nutr 2000; 19: 478-86.

[9] Kruger MC, Coetzer H, de Winter R, Gericke G, van Papendrop DH. Calcium, gamma-linolenic acid and eicosapentaenoic acid supplementation in senile osteoporosis. Aging(Milano) 1998; 10: 385-94.

[10] Terano T. Effect of omega-3 polyunsaturated fatty acid ingestion on bone metabolism and osteoporosis. World Rev Nutr Diet 2001; 88: $141-7$.

[11] Michelson D, Stratakis C, Hill L, et al. Bone mineral density in women with depression. N Engl J Med 1996; 335: 1176-81.

[12] Singh RB, Ghosh S, Niaz MA, Rastogi V. Validation of physical activity and socioeconomic status questionnaire in relation to food intakes for the five city study and a proposed clasification for Indians. J Assoc Phys India 1997; 45: 603-7.

[13] Narsinga RBS, Deosthale YG, Pant KC. Nutrient value of Indian foods. National Institute of Nutrition, Indian Council of Medical Research, Hyderabad, India 1989.

[14] Esterbaur H, Cheeseman K. Determination of aldehyde peroxidation products malondialdehyde and 4-hydroxynonenal. Methods Enzymol 1990; 186: 407-21.

[15] Naito C, Kuwamura M, Yamamoto Y. Lipid peroxides as the initiating factor of atherosclerosis. Ann N Y Acad Sci 1993; 676: 2745.

[16] Watkins BA, Li Y, Allen KG, Hoffman WE, Seifert MF. Dietary ratio of $w-6 / w-3$ polyunsaturated fatty acids alters the fatty acid composition of bone compartments and biomarkers of bone formation in rats. J Nutr 2000; 130: 2274-84.

[17] Watkins BA, Shen CL, Allen K, Seifert MF. Dietary w-3 and w-6 polyunsaturates and acetyle salicyclic acid alter ex vivo PGE2 biosynthesis, tissue IGF1 levels and bone morphometry in chicks. J Bone Miner Res 1996; 11: 1321-32.

[18] Atkinson TG, Barker HJ, Mechling GKA. Incorporation of long chain w-3 fatty acids in tissues and enhanced bone marrow cellularity with docosahexaenoic acid feeding in post weaning Fischers. Lipids 1997; 32: 293-302.

[19] Tashjian AH, Voelkel EF, Lazzaro M, Goad D, Bosma T, Levine L. Tumor necrosis factor-alpha(cachectin) stimulates bone reabsorption in mouse calvaria via a prostaglandin mediated mechanism. Endocrinology 1997; 120: 2029-36. 